\title{
Coupled sloshing in hyperbolic containers suspended as a bifilar pendulum.
}

\author{
M. R. Turner \\ Department of Mathematics \\ University of Surrey \\ Guildford, Surrey GU2 7XH \\ UK \\ Patrick Weidman \\ Department of Mechanical Engineering \\ University of Colorado \\ Boulder, CO 80309-0427 \\ USA
}

\begin{abstract}
The coupled interaction between a sloshing fluid in a partially-filled container suspended as a bifilar pendulum is investigated. The sloshing fluid has a free-surface upon which waves are generated this fluid contributes a restoring force to the container motion by its weight through the wire suspensions and the free-surface waves may either enhance or diminish the restoring force through hydrodynamic interaction with the container walls. Results are presented for inviscid, irrotational sloshing in both a two-dimensional hyperbolic container and a threedimensional hyperboloid container. Frequency results for the coupled system are presented for various pendulum lengths and fluid fill heights. It is found that for long pendulum lengths the container and the fluid oscillate in a synchronous motion when the vessel is released with typical experimental initial conditions, but for pendulum lengths below a given threshold the container and fluid oscillate asynchronously from the same initial condition.
\end{abstract}




\section{Introduction}

The study of the coupled sloshing dynamics of containers partially-filled with a fluid is of practical importance in many physical applications. Unlike situations where the sloshing motion is bought on by the prescribed motion of the vessel, the coupled problem, where the vessel motion depends on the fluid motion within, brings a new dimension which can increase or decrease the amplitude of the sloshing motion. This problem is of practical interest in situations such as the transport of liquids along roads, maritime fluid transport and the dynamics of the sloshing fuel in rockets and aircraft. A theory for the horizontal oscillations of a beam with a liquid-containing cavity was reported by Moiseyev (1964) and other problems of this type related to space vehicle technology may be found in a NASA publication edited by Abramson (1966). The works of Moiseyev \& Rumyantsev (1968), Ibrahim (2005) and Faltinsen \& Timokha (2009), and the references herein, highlight many other related problems to coupled sloshing.

Of interest in this paper is Cooker's sloshing experiment (Cooker, 1994). In this experiment a rectangular container partially-filled with fluid is suspended as a bifilar pendulum by suspension cables of equal length $l$. The container is set into a swinging motion in which the base of the tank remains horizontal throughout its motion so that the fluid motion can be considered as irrotational. This experiment is in the spirit of those conducted by Taylor (1974), i.e. it is simple and easy to construct and yet highlights a fundamental question in fluid mechanics, here being the question of fluid-container interaction. For small initial displacements of the container, such that the container motion is approximately horizontal, Cooker found that after some initial transient sloshing motion, the system settled down to

periodic oscillations with frequency $\omega_{S}<\omega_{0}$ where $\omega_{0}=\sqrt{g / l}$ is the frequency of the dry container, and $g$ is the gravitational constant. In these oscillations the system was synchronous and the fluid in-phase with the pendulum while for other initial conditions the system was asynchronous, with the fluid motion out-of-phase with the container. In this case the frequency of the container was $\omega_{A}$, such that $\omega_{S}<\omega_{A}$.

Cooker developed a linear theory to explain the above observations using an inviscid shallow-water model which assumes that the pressure on the container walls used to calculate the restoring force of the fluid is hydrostatic. The resulting transcendental characteristic equation for the system frequencies was solved numerically and the resulting frequency spectra was in very good agreement with the experiments. Cooker also formulated the theory for a more general two-dimensional hyperbolic geometry by considering linear potential theory 
for the fluid, but with the hydrostatic pressure assumption. Both synchronous and asynchronous frequencies of a planar free-surface oscillating about its central nodal line were reported, again showing that $\omega_{S}<\omega_{A}$. The limiting form of the container, a wedge with $90^{\circ}$ vertex angle, was also considered.

Weidman \& Turner (2016) presented, inter alia, experimental results on the motion of the $90^{\circ}$ wedge suspended as a bifilar pendulum, and found that the hydrostatic pressure assumption of Cooker (1994) underestimated the synchronous frequencies measured. Using full potential theory for which the hydrostatic pressure assumption is removed, they obtained excellent agreement between theory and experiments. Although Cooker derived the characteristic equation for the frequencies of motion of the hyperbolic container, albeit using the hydrostatic assumption, he did not investigate the interesting behaviours inherent in the system. Roberts (2005) partially formulated the potential theory for the hyperbolic container, but focussed on an analysis for the limiting case of a $90^{\circ}$ wedge. In the current paper we present the corresponding characteristic equation for the non-hydrostatic pressure assumption, and investigate features of its solution for both the two-dimensional hyperbolic container and the equivalent three-dimensional hyperboloid container.

The theory for oscillations of a container suspended as a bifilar pendulum executing small sideways oscillations reveals two fundamental parameters. One is the ratio of fluid mass $m$ to container mass $m_{0}$ given by $M=m / m_{0}$ and the other is the pendulum length $l$. Indeed, the suspended bifilar sloshing problem has received much attention since the publication of Cooker (1994). Yu (2010) extended the shallow-water theory for the rectangular and cylindrical container to the non-shallow-water scenario and found the eigenmodes for these problems consist of the shallow-water eigenmode plus a sum of vertical eigenmodes (Linton and McIver, 2001). Results were presented showing the dramatic effect of non-shallow fluid depths. Alemi Ardakani et al. (2012) demonstrated a 'resonance' effect in the rectangular container system, where anti-symmetric fluid eigenmodes, which couple to the container motion, can have the same oscillation frequency as the symmetric fluid eigenmodes, which exhibit zero force on the container. Weidman \& Turner (2016) also conducted experiments on multi-compartment rectangular containers and showed that measured frequencies were in good agreement with those obtained from the baffled container theory of Turner et al. (2013). Herczynski \& Weidman (2012) produced potential flow theory and experiments for the freesloshing motion $(l \rightarrow \infty)$ of fluid-filled boxes, cylinders, wedges, cones, and cylindrical annuli containers. In this case the sole driving force is the fluid sloshing against the container walls.

Weidman \& Turner (2016) also investigated the initial value problem for both rectangular 
and cylindrical containers suspended as bifilar pendulums. That study was motivated by experimental results showing that the frequency of container oscillations switch to higher frequency modes as $M \rightarrow 0$. The investigation highlighted the importance of considering the initial-value problem in order to fully understand experimental results. The initial-value problems for the hyperbolic and hyperboloid containers are also considered here. We find that using initial conditions readily obtained in laboratory experiments, i.e. simply pulling back the container from equilibrium and releasing it gently with the fluid quiescent, both synchronous and asynchronous motions are possible depending upon the pendulum length $l$.

The presentation is laid out as follows. In $\S 2$ we present the potential theory for a vessel suspended as a bifilar pendulum, with the characteristic frequency equation derived for the $2 \mathrm{D}$ hyperbolic container in $\S 3$ and the $3 \mathrm{D}$ hyperboloid container in $\S 4$. Numerical results are presented in $§ 5.1$ for a fixed vessel geometry which is partially filled to different fluid levels. Section 5.2 presents the corresponding initial value problem and investigates the transition from synchronous to asynchronous sloshing. A summary, discussion and concluding remarks are given in $\S 6$.

\section{Potential flow theory}

We take a coordinate system attached to the container suspended as a bifilar pendulum, as shown in the schematic diagram in figure 1, with the origin $O$ placed at the centreline of the vessel on the quiescent free-surface. The container is moving horizontally with periodic motion $X(t)=X_{0} \cos \omega t$ of frequency $\omega$ in the inertial system with respect to the origin $O^{*}$. The free-surface of the container is located at $z=0$ symmetrically placed with respect to the sidewalls $S$. In what follows, we use the notation and formulation of Faltinsen \& Timokha $(2009, \S 2.4 .2)$. The potential function $\Phi(x, y, z, t)$ for linear motion of an inviscid, incompressible, irrotational fluid in container $V$ must satisfy Laplace's equation

$$
\nabla^{2} \Phi=0 \quad(\text { in } V)
$$

This equation is solved with the kinematic free-surface condition

$$
\eta_{t}=\Phi_{z}, \quad(\text { on } z=0)
$$

and the dynamic free-surface condition

$$
\Phi_{t}+g \eta+x \ddot{X}=0, \quad(\text { on } z=0)
$$


where $\eta(x, y, t)$ is the free-surface displacement and $g$ is the gravitational constant, along with the impermeability condition on the container walls

$$
\mathbf{n} \cdot \boldsymbol{\nabla} \Phi=0, \quad(\text { on } S)
$$

where $\mathbf{n}$ is the direction of the outward unit normal. Note here that the subscripts $t$ and $z$ denote partial derivatives with respect to these variables, and the over-dot represents a full derivative with respect to $t$.

The motion of the container is governed by Newton's second law of motion

$$
m_{0} a_{x}=F_{R}+F_{H}
$$

where $m_{0}$ is the mass of the container, $a_{x}=\ddot{X}(t)$ is its horizontal acceleration, $F_{R}$ is the restoring force caused by the pendulum displacement, and $F_{H}$ is the hydrodynamic force of the fluid acting on the container side-wall in the direction of the positive $x$-axis. For small displacements

$$
F_{R}=-\left(m+m_{0}\right) \frac{g X(t)}{l}=-\left(m+m_{0}\right) \omega_{0}^{2} X(t)
$$

where $m$ is the mass of liquid in the container, $l$ is the length of the pendulum supports, and $\omega_{0}=\sqrt{g / l}$ is the frequency of oscillation of a dry container. Also

$$
F_{H}=\int_{S} p(S)(\mathbf{n} \cdot \mathbf{i}) d S
$$

where $\mathbf{i}$ is the unit vector in the direction of the positive $x$-axis and $p(S)$ is the potential pressure in the moving coordinate system on the container wall given as

$$
p(S)=-\rho\left(\Phi_{t}+g z+x \ddot{X}\right)
$$

where $S$ is the quiescent wetted surface, i.e. the still-water wetted boundary below $z=0$.

For both containers we assume the free-surface profile

$$
\eta(x, t)=\frac{\eta_{0}}{H} x \cos \omega t
$$

where $x$ is used for the two-dimensional hyperbolic container and $x=r \cos \theta$ is used for the hyperboloid container, $\eta_{0}$ is the amplitude of wave displacement, and $H$ is the maximum depth of the containers. Then Eq. (2.1b) becomes

$$
\Phi_{z}=-\frac{\eta_{0} \omega}{H} x \sin \omega t
$$


and suggests a solution of Laplace's equation given by

$$
\Phi(x, y, z, t)=-\frac{\eta_{0} \omega}{H} x z \sin \omega t+F(x, y, t)
$$

which requires $F_{x x}=0$ giving $F(x, y, t)=x F_{1}(y, t)+F_{2}(y, t)$.

Combining the dynamic and kinematic free-surface conditions $(2.1 \mathrm{~b}, \mathrm{c})$ gives

$$
\Phi_{t t}+g \Phi_{z}+x \dddot{X}=0 \quad(\text { on } z=0)
$$

and inserting the posited solution (2.7) yields

$$
x\left[F_{1 t t}-\frac{g \eta_{0} \omega}{H} \sin \omega t+\dddot{X}\right]+F_{2 t t}=0 \quad(\text { on } z=0) .
$$

This provides two condition equations, one yielding $F_{2}(y, t)=a(y)+b(y) t$. Finite solutions are obtained for $b=0$ and since we expect $\Phi$ to be independent of $y, a$ just gives a constant shift in $\Phi$ so it is also set equal to zero. Integration of the term in brackets gives

$$
F_{1}(y, t)=-\frac{g \eta_{0}}{H \omega} \sin \omega t-\dot{X}+c(y) t+d(y)
$$

and for the same reason as for $F_{2}(y, t)$ we take $c=d=0$. For the assumed periodic container motions $X(t)=X_{0} \cos \omega t$ this furnishes the potential function

$$
\Phi(x, z, t)=x\left[X_{0} \omega-\frac{g \eta_{0}}{H \omega}-\frac{\eta_{0} \omega}{H} z\right] \sin \omega t
$$

where the reader is reminded that the pre-multiplier is taken as the Cartesian coordinate $x$ for the hyperbolic container and as cylindrical coordinates $r \cos \theta$ for the hyperboloid container. At this point in the development we pursue the analysis separately for the two containers.

\section{The hyperbolic container}

For the quasi-two-dimensional container we take the container wall profile as $z=-h(x)$ and assume the cross-section is uniform in the $y$-direction, between plane vertical walls of separation width $W$. Inserting this into the impermeability condition (2.1d) gives

$$
\frac{d h}{d x}=-\left[\frac{\Phi_{z}}{\Phi_{x}}\right]_{z=-h(x)} .
$$

Using (2.11), simplifying, and separating variables provides the equation for the container shape

$$
\left(h(x)-\frac{g}{\omega^{2}}+\frac{X_{0} H}{\eta_{0}}\right) d h=x d x
$$


Upon integration we follow Roberts (2005) and set the quiescent free-surface $h=0$ at $x= \pm c$ to obtain

$$
h^{2}+2\left(\frac{X_{0} H}{\eta_{0}}-\frac{g}{\omega^{2}}\right) h=x^{2}-c^{2} .
$$

This gives the container profile $z=-h(x)$ as the hyperbola

$$
z(x)=-Q+\sqrt{x^{2}+Q^{2}-c^{2}}
$$

where

$$
Q=\left(\frac{g}{\omega^{2}}-\frac{X_{0} H}{\eta_{0}}\right)
$$

in which $|c| \leq Q$. We find $Q$ in terms of the geometry of the hyperbola by setting $z=-H$ (the maximum fluid depth) at $x=0$ giving

$$
Q=\frac{H^{2}+c^{2}}{2 H}
$$

The sidewalls of the hyperbolic container have slope \pm 1 as $x \rightarrow \pm \infty$ showing the asymptotes tend to a $90^{\circ}$ wedge with apex at $z=-c$ on the centreline.

The volume of the liquid in a hyperbolic container of width $W$, length $2 c$ and depth $H$ is given as

$$
V=W \int_{-c}^{c} \int_{-h(x)}^{0} d x d z
$$

In the sequel we define $K^{2}=Q^{2}-c^{2}$ to find $h(x)$ and its derivative, viz.

$$
h(x)=Q-\sqrt{K^{2}+x^{2}}, \quad \frac{d h}{d x}=-\frac{x}{\sqrt{K^{2}+x^{2}}}
$$

and thus the volume is given as

$$
V(c, Q)=W\left[c Q-\frac{K^{2}}{2} \ln \left(\frac{Q+c}{Q-c}\right)\right] .
$$

We write $V=V(c, Q)$ rather than $V=V(c, H)$ as it is more convenient for subsequent analysis. The value of $Q$ is related to $H$ and $c$ via (3.5).

We are now in a position to calculate the horizontal hydrodynamic force $F_{H}$ of the liquid acting on the container using (2.4a). Evaluating the potential pressure in $(2.4 \mathrm{~b})$ at the container wall, given by (3.4), provides the expression

$$
F_{H}=\rho W \int_{-c}^{c}\left[\left(\frac{\eta_{0} g}{H}-\frac{\eta_{0} \omega^{2}}{H} h(x)\right) x \cos \omega t+g h(x)\right]\left(-\frac{d h}{d x}\right) d x
$$


wherein the last term integrates to zero. Writing the remaining terms as

$$
F_{H}=-\rho\left(\frac{\eta_{0} g}{H} I_{1}(c, Q)-\frac{\eta_{0} \omega^{2}}{H} I_{2}(c, Q)\right) \cos \omega t,
$$

where

$$
I_{1}(c, Q)=W \int_{-c}^{c} x \frac{d h}{d x} d x, \quad I_{2}(c, Q)=W \int_{-c}^{c} x h \frac{d h}{d x} d x
$$

and integrating provides the simple relations

$$
I_{1}(c, Q)=-V(c, Q), \quad I_{2}(c, Q)=-Q V(c, Q)+\frac{2}{3} W c^{3} .
$$

Thus the periodic horizontal force is

$$
F_{H}=\frac{\eta_{0}}{H}\left[g m+\left(\chi m_{0}-Q m\right) \omega^{2}\right] \cos \omega t
$$

where $m=\rho V(c, Q)$ is the mass of liquid in the container and $\chi=2 \rho W c^{3} /\left(3 m_{0}\right)$. Inserting this into Newton's second law (2.2) yields

$$
m_{0} X_{0} \omega^{2}=\left(m+m_{0}\right) \omega_{0}^{2} X_{0}-\frac{\eta_{0}}{H}\left[g m+\left(\chi m_{0}-Q m\right) \omega^{2}\right] .
$$

Dividing by $m_{0}$ and introducing the dimensionless liquid mass

$$
M=\frac{m}{m_{0}}
$$

and subsequent rearrangement gives the amplification ratio

$$
\frac{\eta_{0}}{X_{0}}=\frac{H\left[(M+1) \omega_{0}^{2}-\omega^{2}\right]}{\left[M g+(\chi-Q M) \omega^{2}\right]} .
$$

Another relation for the amplification ratio obtained from Eq. (3.4b) is

$$
\frac{\eta_{0}}{X_{0}}=\frac{H \omega^{2}}{g-Q \omega^{2}}
$$

Equating these expressions furnishes the quartic equation for the frequency of pendulum motion

$$
(Q(1+M)-\chi) \omega^{4}-(1+M)\left(Q \omega_{0}^{2}+g\right) \omega^{2}+(1+M) g \omega_{0}^{2}=0 .
$$

One check on the derivation of this equation is to consider the $90^{\circ}$ wedge limit in which $H=c$, for which $Q=H$ and $K^{2}=0$. This provides the eigenvalue equation for periodic motion of a bifilar suspended wedge, viz.

$$
\left(1+\frac{M}{3}\right) \omega^{4}-(1+M)\left(\omega_{0}^{2}+\frac{g}{H}\right) \omega^{2}+(1+M) \frac{g}{H} \omega_{0}^{2}=0
$$


in agreement with the result reported in Weidman \& Turner (2016).

Note that varying either $c$ or $Q$ alters the shape of the container, but in an experiment one would likely have a fixed container with different levels of fill. Considering now a fixed container shape given by $c=c^{*}, H=H^{*}$ and $Q=Q^{*}$, partial fillings in the fixed container are found by varying $c$ in (3.16) with $Q \equiv Q(c)$ given by

$$
Q(c)=\frac{\left(H^{*}-Q^{*}\right)^{2}+2 c^{2}+\left(Q^{* 2}-c^{* 2}\right)+2\left(H^{*}-Q^{*}\right) \sqrt{Q^{* 2}-c^{* 2}+c^{2}}}{2\left(H^{*}-Q^{*}+\sqrt{Q^{* 2}-c^{* 2}+c^{2}}\right)}
$$

and with $M \equiv M(c)$ defined as

$$
M(c)=\frac{\rho W}{m_{0}}\left[c Q(c)-\frac{K(c)^{2}}{2} \ln \left(\frac{Q(c)+c}{Q(c)-c}\right)\right]
$$

where

$$
K(c)^{2}=Q(c)^{2}-c^{2} .
$$

This approach implies that for each value of $c$ the geometry of the container remains unchanged, and the depth of the fluid, whose free-surface lies between $x= \pm c$, is given by

$$
H=H^{*}-h(c)=H^{*}-Q^{*}+\sqrt{K^{* 2}+c^{2}} .
$$

Note that $Q(c)$ in (3.18) is determined from the form of $Q$ in (3.5) with $H$ given by (3.20).

Sample profiles showing the depth variation at $c=\{0.25,0.5,0.75,1.0\} \mathrm{m}$ for a fixed container defined by $c^{*}=1.0 \mathrm{~m}$ and $H^{*}=0.5 \mathrm{~m}$ are displayed in figure 2 . The respective fluid depths in these containers are $H=\{0.040569,0.151388,0.310600,0.500000\} \mathrm{m}$.

\section{The hyperboloid container}

We now turn attention to the axisymmetric hyperboloid container suspended as a bifilar pendulum in which case we take the container wall at $z=-h(r)$ and the free-surface lies between $r \in[0, R]$. The solution for the container shape follows exactly that in the preceding section: one simply replaces $x$ by $r$ and $c$ by $R$ in Eq. (3.4a) to obtain

$$
z(r)=-Q+\sqrt{r^{2}+K^{2}}
$$

with $Q$ again given by Eq. (3.4b) but now $K^{2}=Q^{2}-R^{2}$. Setting the deepest point $z=-H$ at $r=0$ gives $Q$ as

$$
Q=\frac{H^{2}+R^{2}}{2 H}
$$


The sidewalls of the hyperboloid has slopes \pm 1 for $r \rightarrow \pm \infty$ showing the asymptotes tend to a $90^{\circ}$ cone with apex at $z=-R$ on the centreline. The volume of the hyperboloid is given as

$$
V(R, Q)=\int_{0}^{R} \int_{-h(r)}^{0} r d r d \theta d z=\pi H\left(\frac{R^{2}}{2}-\frac{H^{2}}{6}\right)=\frac{\pi}{3}\left(3 Q R^{2}-2 Q^{3}+2\left(Q^{2}-R^{2}\right)^{3 / 2}\right)
$$

the second form of which, although more complicated, is convenient for examining results when considering a partially filled hyperboloid container with a fixed geometry.

The horizontal hydrodynamic force $F_{H}$ of the liquid acting on the hyperboloid is now calculated. Evaluating the potential pressure in (2.4b) at the container wall given by (4.1) provides the result

$$
F_{H}=\rho \int_{0}^{R} \int_{0}^{2 \pi}\left[\left(\frac{\eta_{0} g}{H}-\frac{\eta_{0} \omega^{2}}{H} h(r)\right) r \cos \theta \cos \omega t+g h(r)\right]\left(-\frac{d h}{d r}\right) r \cos \theta d \theta d r
$$

the last term of which integrates to zero. Writing the remaining terms as

$$
F_{H}=-\rho \pi\left(\frac{\eta_{0} g}{H} J_{1}-\frac{\eta_{0} \omega^{2}}{H} J_{2}\right) \cos \omega t
$$

subsequent integration gives

$$
J_{1}(R, Q)=-\frac{V(R, Q)}{\pi}, \quad J_{2}(R, Q)=-\frac{Q V(R, Q)}{\pi}+\frac{R^{4}}{4}
$$

with $V(R, Q)$ as defined in (4.3). The equation for the hydrodynamic force is thus given exactly by (3.11) but here $\chi=\rho \pi R^{4} /\left(4 m_{0}\right)$. Therefore the analysis of $\S 3$ can be copied here giving the same quartic equation (3.16) for the frequency of the vessel.

In the limiting case of a $90^{\circ}$ cone for which $R=Q=H$ one finds the quartic equation for the frequency given as

$$
\left(1+\frac{M}{4}\right) \omega^{4}-(1+M)\left(\frac{g}{l}+\frac{g}{H}\right) \omega^{2}+(1+M) \frac{g^{2}}{l H}=0
$$

where the substitution $\omega_{0}^{2}=g / l$ has been used. In the limit of infinite pendulum length $l \rightarrow \infty$ we recover the frequency for a container driven by liquid sloshing reported by Herczynski and Weidman (2012), viz.

$$
\frac{\omega}{\omega_{R}}=\frac{1}{M^{1 / 6}} \sqrt{\left(\frac{1+M}{1+\frac{M}{4}}\right)}
$$


where the reference frequency for free container motion is

$$
\omega_{R}=\left(\frac{\rho \pi g^{3}}{3 m_{0}}\right)^{1 / 6}
$$

As for the hyperbolic container, varying $Q$ or $R$ modifies the container shape, but we can consider a partially filled hyperboloid by choosing $Q(R)$ accordingly. For a fixed hyperboloid container given by $R=R^{*}, H=H^{*}$ and $Q=Q^{*}$, we again consider the frequency of partial fillings in this container by varying $R$ in (4.9) with $Q \equiv Q(R)$ given by

$$
Q(R)=\frac{\left(H^{*}-Q^{*}\right)^{2}+2 R^{2}+\left(Q^{* 2}-R^{* 2}\right)+2\left(H^{*}-Q^{*}\right) \sqrt{Q^{* 2}-R^{* 2}+R^{2}}}{2\left(H^{*}-Q^{*}+\sqrt{Q^{* 2}-R^{* 2}+R^{2}}\right)}
$$

and with $M \equiv M(R)$ given as

$$
M(R)=\frac{\rho \pi}{3 m_{0}}\left[Q(R)\left(3 R^{2}-2 Q(R)^{2}\right)+2\left(Q(R)^{2}-R^{2}\right)^{3 / 2}\right] .
$$

In this case the depth of the fluid which lies between $r=[0, R]$ for $\theta=[0,2 \pi]$ is

$$
H=H^{*}-h(r)=H^{*}-Q^{*}+\sqrt{K^{* 2}+R^{2}} .
$$

\section{Results for a fixed container geometry}

For all results presented in this section we take the width of the two-dimensional hyperbolic tank $W=0.5 \mathrm{~m}$ and both tank masses $m_{0}=10 \mathrm{~kg}$.

\subsection{Frequency behaviour}

The quartic equation (3.16) has two positive solutions given by

$$
\begin{aligned}
& \omega_{S}=\omega_{0} \sqrt{\frac{(1+M)(Q+l)-\sqrt{(1+M)^{2}(Q-l)^{2}+4 l(1+M) \chi}}{2(Q(1+M)-\chi)}}, \\
& \omega_{A}=\omega_{0} \sqrt{\frac{(1+M)(Q+l)+\sqrt{(1+M)^{2}(Q-l)^{2}+4 l(1+M) \chi}}{2(Q(1+M)-\chi)}},
\end{aligned}
$$

where $\chi=2 \rho W c^{3} /\left(3 m_{0}\right)$ for the 2D hyperbolic container and $\chi=\rho \pi R^{4} /\left(4 m_{0}\right)$ for the 3D hyperboloid container. From $(2.5)$ we know that the free-surface amplitude is given by $\eta_{0} / H$ which from (3.15) gives

$$
\frac{\eta_{0}}{H}=\frac{\omega^{2} X_{0}}{g-Q \omega^{2}}
$$


Thus when the product of $\eta_{0} / H$ with $X_{0}$ is positive the free-surface and the container motion are said to be synchronous or in-phase (denoted by $S$ in (5.1)), and when negative they are said to be asynchronous or out-of-phase (denoted by $A$ in (5.1)). The correct labelling of the frequencies above for the \pm square root cases can easily be seen, by considering the denominator $g-Q \omega^{2}$ which ultimately determines the sign of $X_{0} \eta_{0}$ according to

$$
\begin{array}{r}
g-Q \omega^{2}=-\frac{\omega_{0}^{2}}{2(Q(1+M)-\chi)}[Q(1+M)(Q-l)+2 l \chi \\
\left. \pm Q \sqrt{(1+M)^{2}(Q-l)^{2}+4 l(1+M) \chi}\right] .
\end{array}
$$

One can show that

$$
Q \sqrt{(1+M)^{2}(Q-l)^{2}+4 l(1+M) \chi} \geq Q(1+M)(Q-l)+2 l \chi,
$$

for all $c$ or $R$ where one needs to know that $Q(1+M)-\chi \geq 0$ for all $c$ or $R$. This can easily be deduced by noting that the hyperbolic container is bounded above by the $90^{\circ}$ wedge container with depth $c^{*}$ and below by the $90^{\circ}$ wedge container with depth $H$ (a similar argument holds for the hyperboloid being bounded by two $90^{\circ}$ cones); consequently we find

$$
0 \leq H\left(1+\frac{M}{3}\right) \leq Q(1+M)-\frac{2}{3} \frac{\rho W c^{3}}{m_{0}} \leq c^{*}\left(1+\frac{M}{3}\right) .
$$

Thus from (5.3) the positive square root leads to $X_{0} \eta_{0}<0$ and vice-versa for the negative root. One should also note that (5.2) suggests that resonance occurs in the system when $\omega^{2}=g / Q=l \omega_{0}^{2} / Q$, but substitution into (3.16) leads to the conclusion that this frequency occurs only when $\chi=0$, i.e., it does not occur for a finite liquid mass.

Frequencies for the fixed containers $c^{*}=R^{*}=1 \mathrm{~m}, H^{*}=0.5 \mathrm{~m}$, and $l=0.5 \mathrm{~m}$ and $l=2.0 \mathrm{~m}$ are plotted as a function of $M$ in figure $3 \mathrm{a}$ for the hyperbolic container and in figure $3 \mathrm{~b}$ for the hyperboloid container. These show that the synchronous frequency always decreases from its initial value at $M=0$, while the asynchronous frequency increases from its $M=0$ value to some maximum value before decreasing again, but always satisfies $\omega_{S} \leq \omega_{A}$.

Cooker (1994) derived the quartic equation for the frequencies of the hyperbolic container, but with the caveat that the pressure was hydrostatic. From his equation we find the synchronous and asynchronous frequencies to be

$$
\omega_{S}^{C}=\frac{\omega_{0}}{\sqrt{2 Q}} \sqrt{(1+M)(Q+l)-\sqrt{(1+M)^{2}(Q+l)^{2}-4 Q l(1+M)}}
$$




$$
\omega_{A}^{C}=\frac{\omega_{0}}{\sqrt{2 Q}} \sqrt{(1+M)(Q+l)+\sqrt{(1+M)^{2}(Q+l)^{2}-4 Q l(1+M)}} .
$$

These results are compared with our potential theory results $(5.1)$ in figure 4 for $c^{*}=1 \mathrm{~m}$, $H^{*}=0.5 \mathrm{~m}$, and $l=2 \mathrm{~m}$. It is clear that the hydrostatic assumption underestimates the synchronous frequency $\omega_{S}$ as $M$ increases, while the asynchronous frequency is greatly overestimated by the hydrostatic assumption, the same conclusion as for $90^{\circ}$ wedge reported by Weidman \& Turner (2016).

One interesting feature to note in figure 3 for both the hyperbola and hyperboloid containers is that, when the inequality $l>Q(0)=\left(c^{* 2}-H^{* 2}\right) /\left(2 H^{*}\right)$ is satisfied, then $\omega_{S}=\omega_{0}$ at $M=0$, while when $l<Q(0)$ we have $\omega_{A}=\omega_{0}$ at $M=0$. For a physical experiment we expect $\omega \rightarrow \omega_{0}$ as $M \rightarrow 0$, suggesting that as the fluid mass tends to zero, the motion of the container should be interesting; in particular asynchronous sloshing could be observed with a simple initial condition. This is explored further in the next section. In contradistinction to this behaviour, the switching of modes as $M \rightarrow 0$ does not occur in either the wedge or the cone; indeed in these containers it is always $\omega_{S} \rightarrow \omega_{0}$ as $M \rightarrow 0$, whilst for both the wedge and cone geometries $\omega_{A} \rightarrow \infty$ as $M \rightarrow 0$.

\subsection{The initial value problem}

In a physical experiment, the time evolution of the container and the free-surface will be a linear combination of the synchronous and asynchronous modes with frequencies given in (5.1), namely

$$
\begin{gathered}
X(t)=X_{S} \cos \omega_{S} t+X_{A} \cos \omega_{A} t \\
\eta(x, t)=\frac{X_{S} \omega_{S}^{2}}{g-Q \omega_{S}^{2}} x \cos \omega_{S} t+\frac{X_{A} \omega_{A}^{2}}{g-Q \omega_{A}^{2}} x \cos \omega_{A} t
\end{gathered}
$$

where the coefficients $X_{S}$ and $X_{A}$ are fixed by the initial conditions. The simplest, reproducible, initial condition to consider is

$$
X(0)=\widehat{X}, \quad \eta(x, 0)=0
$$

i.e. releasing the container from a small horizontal displacement with the fluid quiescent. Solving the resulting system of equations leads to

$$
X_{S}=\frac{\widehat{X} \omega_{A}^{2}\left(g-Q \omega_{S}^{2}\right)}{g\left(\omega_{A}^{2}-\omega_{S}^{2}\right)}, \quad X_{A}=-\frac{\widehat{X} \omega_{S}^{2}\left(g-Q \omega_{A}^{2}\right)}{g\left(\omega_{A}^{2}-\omega_{S}^{2}\right)} .
$$

Therefore, in order to predict the time evolution of container motion, we need to consider the respective magnitudes of each mode $X_{S}$ and $X_{A}$ to determine which mode (if any) 
dominates the solution. The analysis above also holds for the hyperboloid container, and as the two containers have similar frequency results (see figure 3) we just consider the hyperbolic container here. Plotting $\left|X_{S} / \widehat{X}\right|$ and $\left|X_{A} / \widehat{X}\right|$ for the results in figure 3 a shows that, for the case $l=2.0 \mathrm{~m}$ in figure $5 \mathrm{a}$, it is the synchronous mode which dominates the solution, and $\omega \rightarrow \omega_{0}$ as $M \rightarrow 0$ as expected. On the other hand, for $l=0.5 \mathrm{~m}$ in figure $5 \mathrm{~b}$ it is the asynchronous mode which dominates, and again $\omega \rightarrow \omega_{0}$ when $M \rightarrow 0$. The interesting result here is that from the experimental initial condition (5.8) it is possible to observe asynchronous sloshing motions in the tank by varying the pendulum length $l$. For the results in figures $3 \mathrm{a}$ and $5 \mathrm{~b}$, when $M=10.10$, figure 6 exhibits the evolution of $X(t)$ given by (5.7a). This shows that the higher-frequency asynchronous mode is detectable, but with its amplitude modulated by the lower-frequency synchronous mode.

Finally, it should be noted that it is possible to have an experimental setup for which the synchronous mode is the dominant mode at small mass ratios $M$ whilst the asynchronous mode dominates at large values of $M$. This is achieved by choosing a pendulum length $l$ for which $l>Q$ initially, but at some larger value of $M, l$ becomes less than $Q$. An example of this for the hyperbolic container in figure $3 \mathrm{a}$ is when $l=1 \mathrm{~m}$, and plots of $\left|X_{S} / \widehat{X}\right|$ and $\left|X_{A} / \widehat{X}\right|$ are given in figure 7. However, when this behaviour occurs the magnitudes $X_{S}$ and $X_{A}$ of the modes are similar. This is because as $l$ decreases, the magnitudes of $X_{S}$ and $X_{A}$ become comparable until they become equal for some large value of $M$, when $l=Q(M)$, and then once $l<Q(0)$ the relative magnitude of the modes diverge again (see figures $5 \mathrm{a}, 7$, and $5 \mathrm{~b}$ respectively). When the mode magnitudes are comparable the container evolution $X(t)$ becomes more complex, such as in figure 8 which shows the $M=5.02$ result from figure 7 . In this case it is expected that experimental measurement of the vessel frequency might be difficult.

While the mode switching behaviour as $M \rightarrow 0$ is not observed for the wedge, it is true that the magnitudes of $X_{S}$ and $X_{A}$ become comparable as $l$ decreases until, for $l<H$, there exists a value of $M$ at which $X_{S}=X_{A}$. This may thus explain why the theoretical wedge frequencies presented in Weidman \& Turner (2016) underestimated the experimentally measured frequencies for short pendulum lengths, because the vessel motion also contained a moderate contribution from the asynchronous mode, thus rendering measurement of the frequencies more difficult. 


\section{Summary, discussion and conclusions}

We examined the coupled sloshing of an inviscid, irrotational fluid in two-dimensional and three-dimensional hyperbolic containers, suspended as bifilar pendula. The two control parameters for the problem are the fluid-to-container mass ratio $M=m / m_{0}$ and the pendulum length $l$. It was found that both containers could exhibit synchronous and asynchronous motions with frequencies related by $\omega_{S}<\omega_{A}$. Upon increasing $M$, the synchronous frequency decreases monotonically from its dry container value, while the asynchronous frequency increases from its dry container value, reaches a maximum, and then decreases for larger $M$. For long pendulum lengths, $l>Q(0)=\left(c^{* 2}-H^{* 2}\right) /\left(2 H^{*}\right)$, we find $\omega_{S} \rightarrow \omega_{0}$ as $M \rightarrow 0$, while for short pendulum lengths, $l<Q(0)$, it is $\omega_{A} \rightarrow \omega_{0}$ as $M \rightarrow 0$. Therefore, the interesting asynchronous system behaviour should be observable in experiments for pendulum lengths shorter than $\left(c^{* 2}-H^{* 2}\right) /\left(2 H^{*}\right)$ for the hyperbolic container and shorter than $\left(R^{* 2}-H^{* 2}\right) /\left(2 H^{*}\right)$ for the hyperboloid container. While both synchronous and asynchronous motions are possible in experiments, intuition suggests that only synchronous modes will be evident with a typical initial condition such as a container released from rest with the fluid stationary.

By considering the initial-value problem for the above setup, it was shown that both types of sloshing motion can be observed via the same initial condition for different pendulum lengths. In this case the container displacement (5.7a) is a superposition of the synchronous and asynchronous modes. By calculating the relative magnitudes of the mode amplitudes $X_{S}$ and $X_{A}$ it was shown that for $l>Q(0),\left|X_{S}\right|>\left|X_{A}\right|$ and synchronous sloshing is observed, whilst for $l<Q(0),\left|X_{S}\right|<\left|X_{A}\right|$ and asynchronous sloshing is observed. It was also shown that there exist pendulum lengths for which $\left|X_{S}\right|>\left|X_{A}\right|$ at small $M$ and $\left|X_{S}\right|<\left|X_{A}\right|$ at large $M$, suggesting a change from synchronous to asynchronous sloshing as the container is filled with fluid. However, in such cases the magnitudes of the modes are comparable and thus the container motion is complex. Hence measurement of the container frequencies for this case may be rendered difficult, especially with the inevitable viscous damping.

Future directions of interest would be to conduct the physical experiments on these containers to observe the synchronous/asynchronous modes for varying pendulum lengths, and also to investigate how these two modes interact in a nonlinear setting. Both these scenarios are left to future studies. 


\section{Acknowledgement}

MRT is grateful to the London Mathematical Society who funded his visit to the University of Colorado, Boulder under their Research in Pairs scheme (Ref 41504). 


\section{References}

Alemi Ardakani, H., Bridges, T. J. and Turner, M. R. 2012 Resonance in a model for Cooker's sloshing experiment, Euro. J. Mech. B/Fluids, 36, 25-38.

Abramson, H. N. 1966 The dynamical behavior of liquids in a moving container. Tech. Rep. SP-106, NASA, Washington, DC.

Cooker, M. J. 1994 Water waves in a suspended container. Wave Motion, 20, 385-395.

Faltinsen, O. M. and Timokha, A. N. 2009 Sloshing. Cambridge University Press (Cambridge).

Herczynski, A. and Weidman, P. 2012 Experiments on the periodic oscillations of free containers driven by liquid sloshing, J. Fluid Mech., 693, 216-242.

Ibrahim, R. A. 2005 Liquid Sloshing Dynamics. Cambridge University Press (Cambridge).

Linton, C. M. and McIver, P. 2001 Handbook of Mathematical Techniques for Wave-Structure Interaction, Chapman \& Hall/CRC, Boca Raton.

Moiseyev, N. N. 1964 Introduction to the theory of oscillations of liquid-containing bodies. Adv. Appl. Mech., 8, 233-289.

Moiseyev, N. N. and Rumyantsev, V. V. 1968 Dynamic Stability of Bodies Containing Fluid. Springer-Verlag (New York).

Roberts, C. 2005 The Coupled Motion of Containers and their Sloshing Liquid Loads. MMath project, University of East Anglia. https://ueaeprints.uea.ac.uk/19896/

Taylor, G. I. (1974) The interaction between experiment and theory in fluid mechanics, Ann. Rev. Fluid Mech. 6 1-17.

Turner, M. R., Bridges, T. J. and Alemi Ardakani, H. 2013 Dynamic coupling in Cooker's sloshing experiment with baffles, Phys. Fluids, 25(10), 112102.

Weidman, P. D. and Turner, M. R. 2016 Experiments on the synchronous sloshing in suspended containers described by shallow-water theory, J. Fluids Struct. (in press).

Yu, J. 2010 Effects of finite water depth on natural frequencies of suspended water tanks, Stud. Appl. Math., 125, 337-391. 


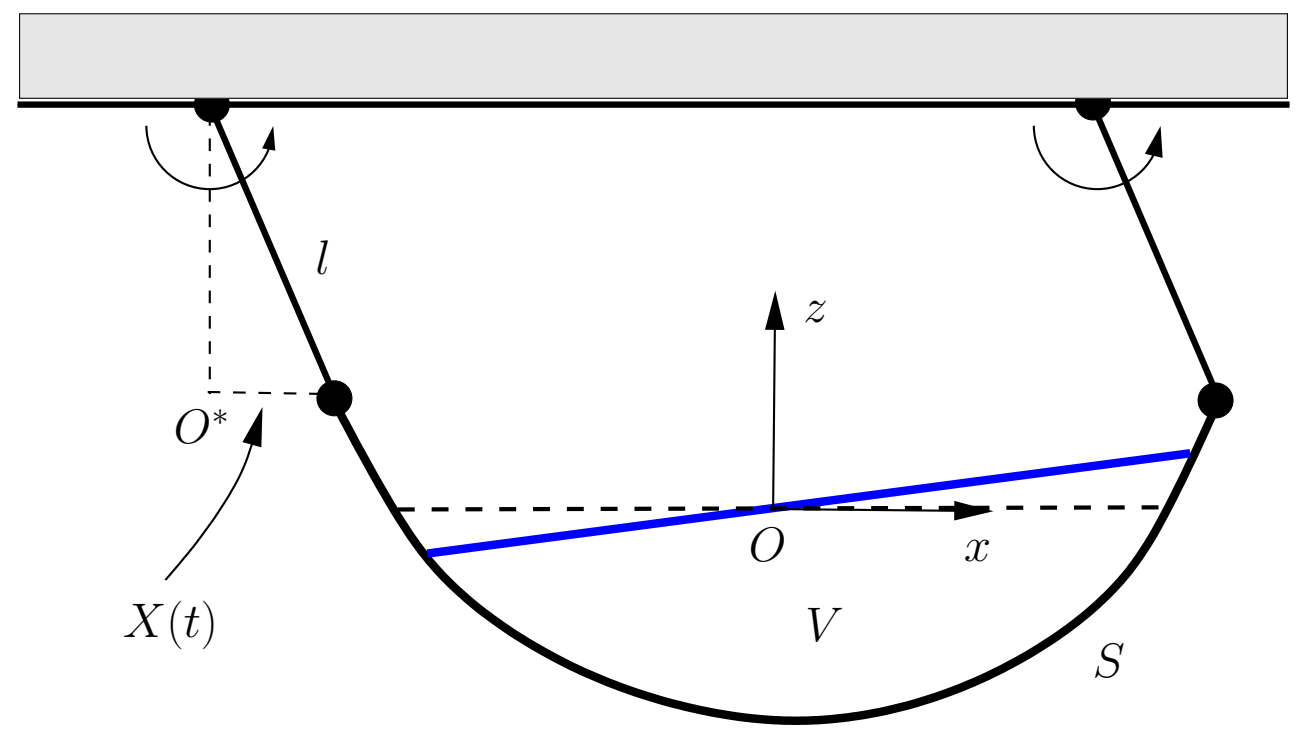

Figure 1. Schematic diagram of hyperbolic container suspended as a bifilar pendulum. In all calculations the mass of each container is take as $m_{0}=10.0 \mathrm{~kg}$.

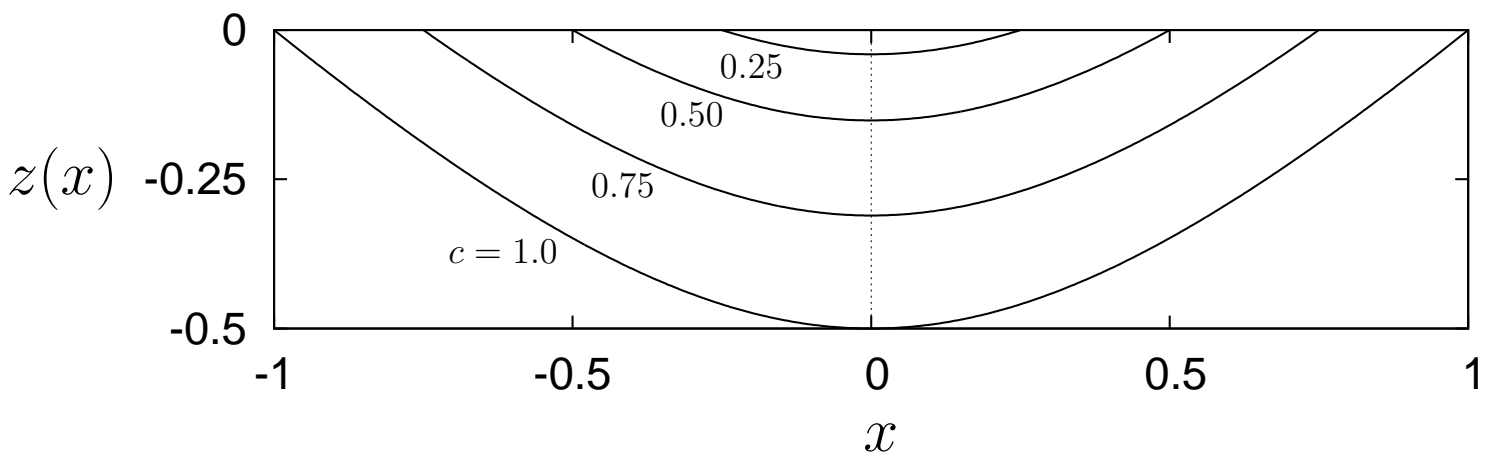

Figure 2. Hyperbolic container profiles for a fixed container defined by $c^{*}=1.0 \mathrm{~m}$ and $H^{*}=0.5 \mathrm{~m}$ plotted for $c=\{0.25,0.5,0.75,1.0\} \mathrm{m}$. The associated depths $H$ are cited in the text. 


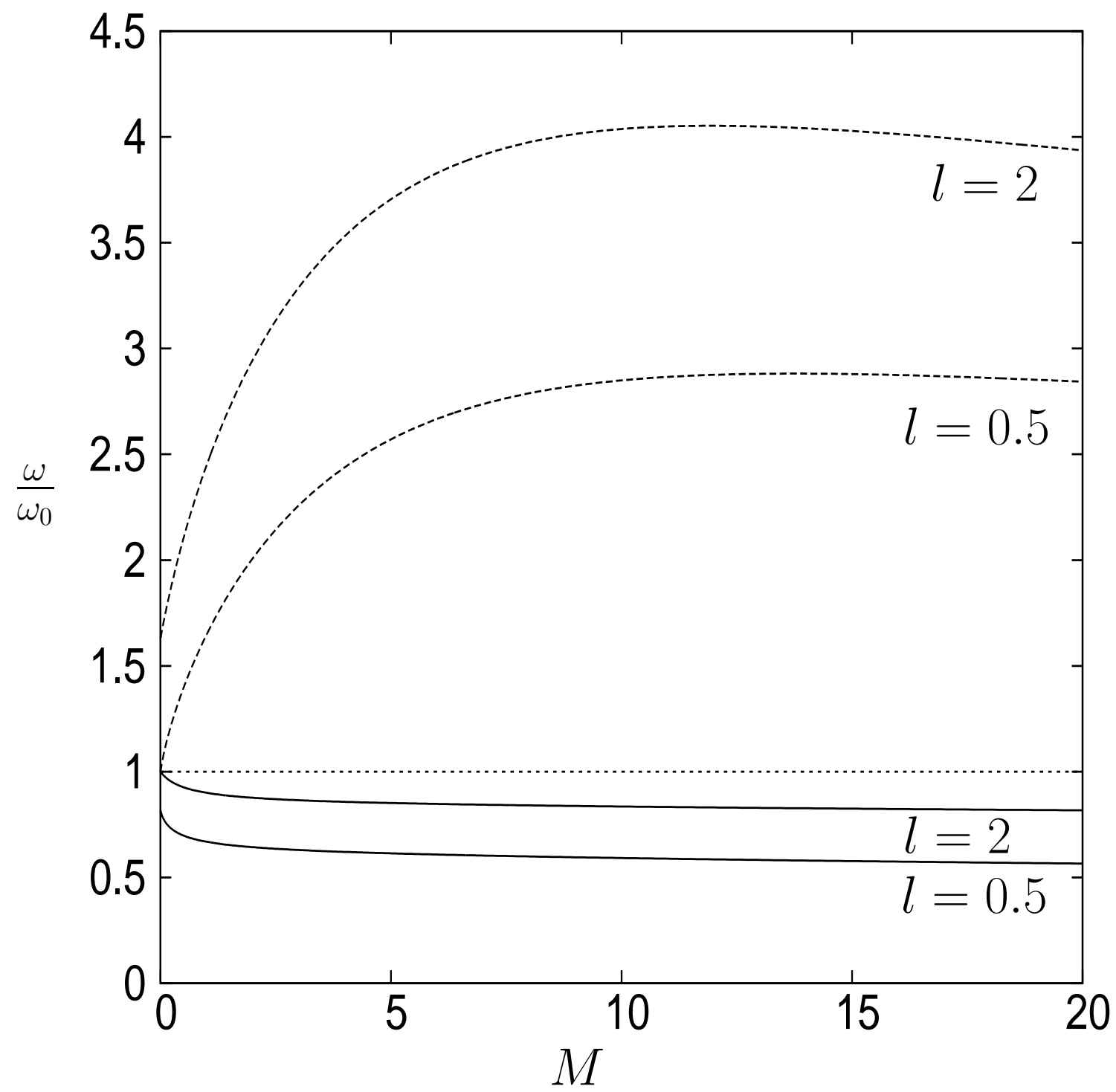

Figure 3a. Plot of $\omega_{S}(M)$ (solid line) and $\omega_{A}(M)$ (dashed line) for the hyperbolic container with $c^{*}=1.0 \mathrm{~m}, H^{*}=0.5 \mathrm{~m}$ with $l=2.0 \mathrm{~m}$ and $l=0.5 \mathrm{~m}$. The dotted line signifies $\omega / \omega_{0}=1$. 


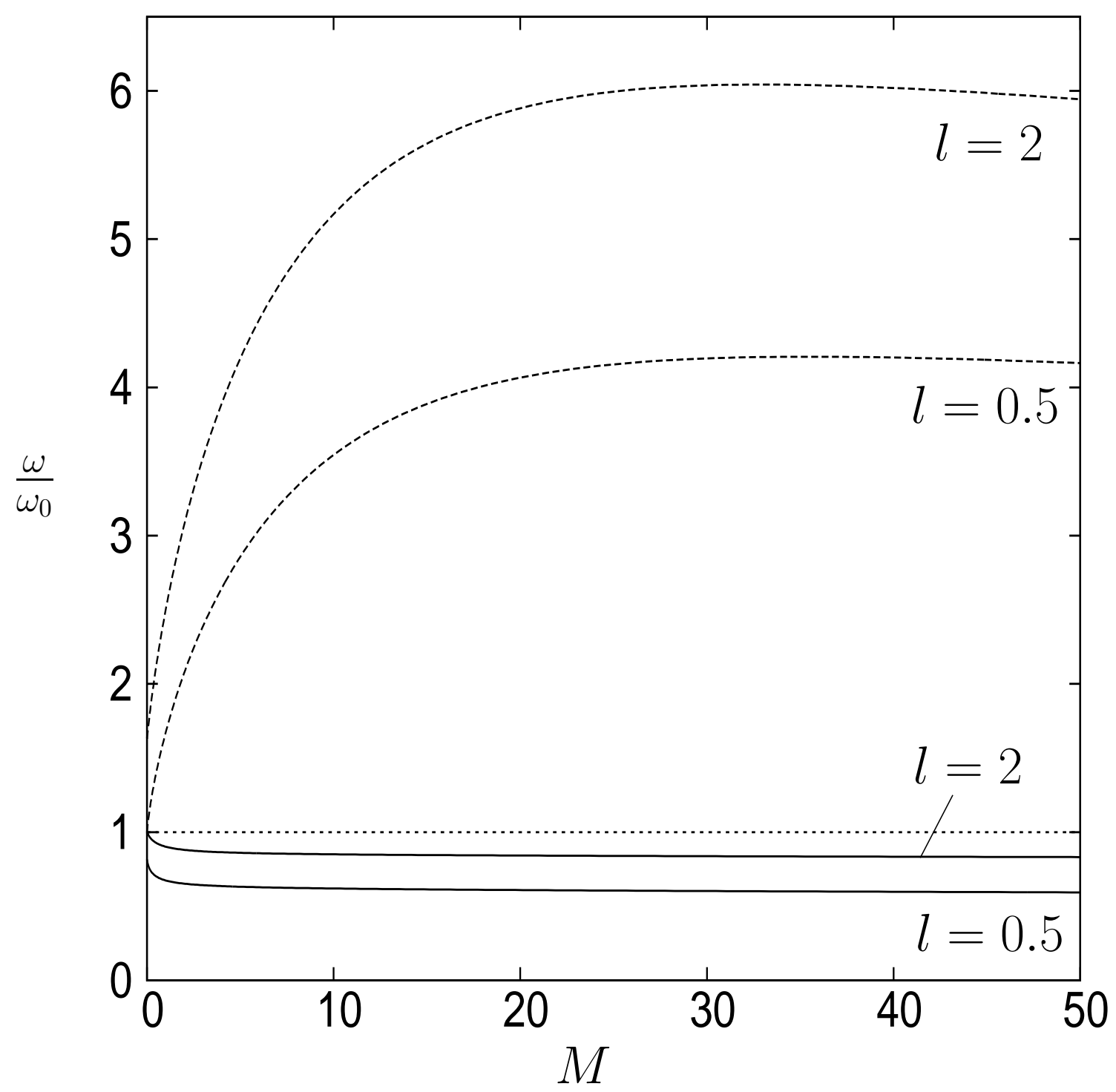

Figure 3b. Plot of $\omega_{S}(M)$ (solid line) and $\omega_{A}(M)$ (dashed line) for the hyperboloid container with $R^{*}=1.0 \mathrm{~m}, H^{*}=0.5 \mathrm{~m}$, with $l=2.0 \mathrm{~m}$ and $l=0.5 \mathrm{~m}$. The dotted line signifies $\omega / \omega_{0}=1$. 


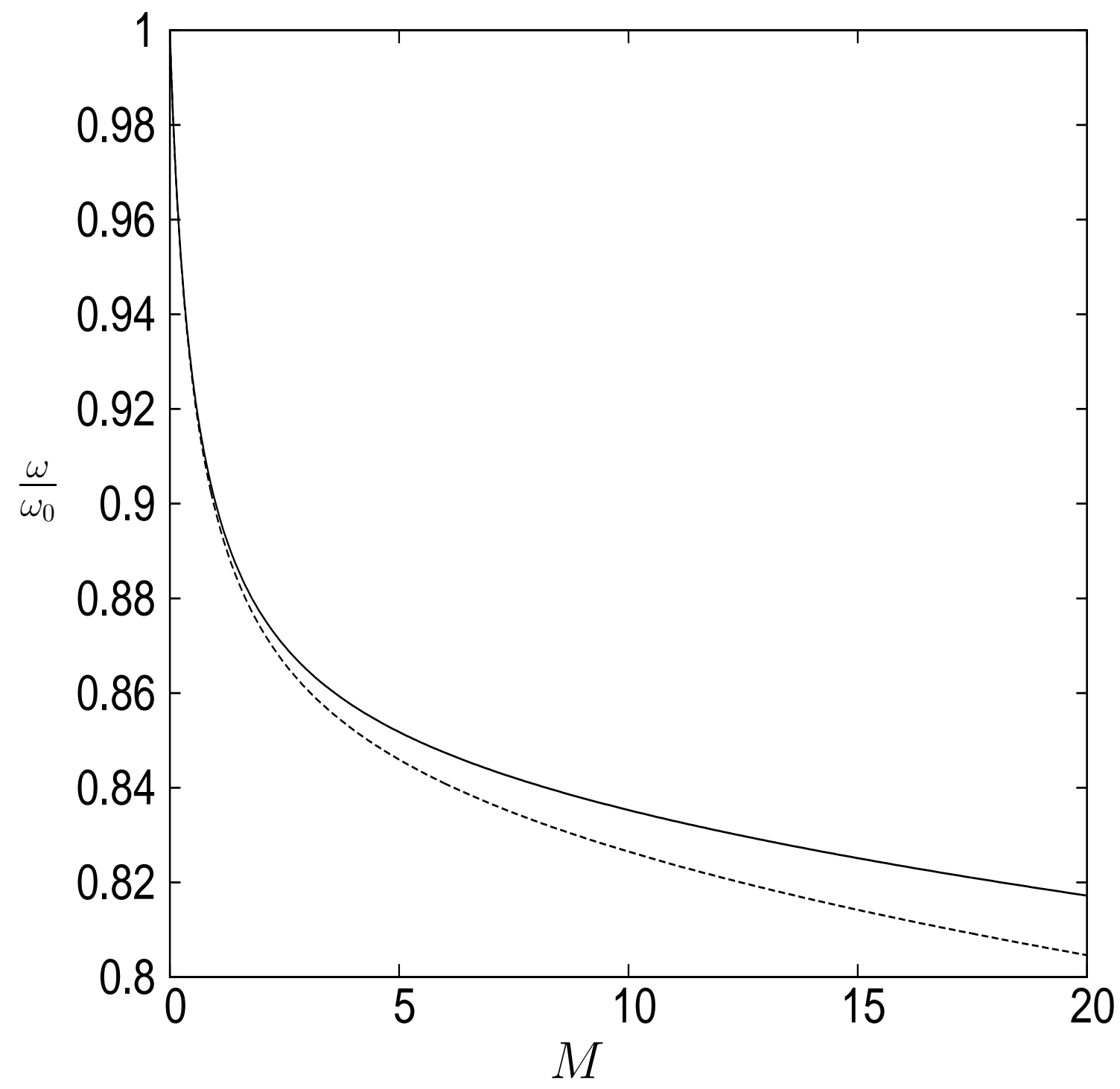

Figure 4a. Plot of $\omega_{S}(M)$ for the hyperbolic container with $c^{*}=1 \mathrm{~m}, H^{*}=0.5 \mathrm{~m}$ and $l=2.0 \mathrm{~m}$ for our non-hydrostatic pressure assumption (5.1) (solid line) and Cooker (1994)'s hydrostatic pressure assumption (5.6) (dashed line). 


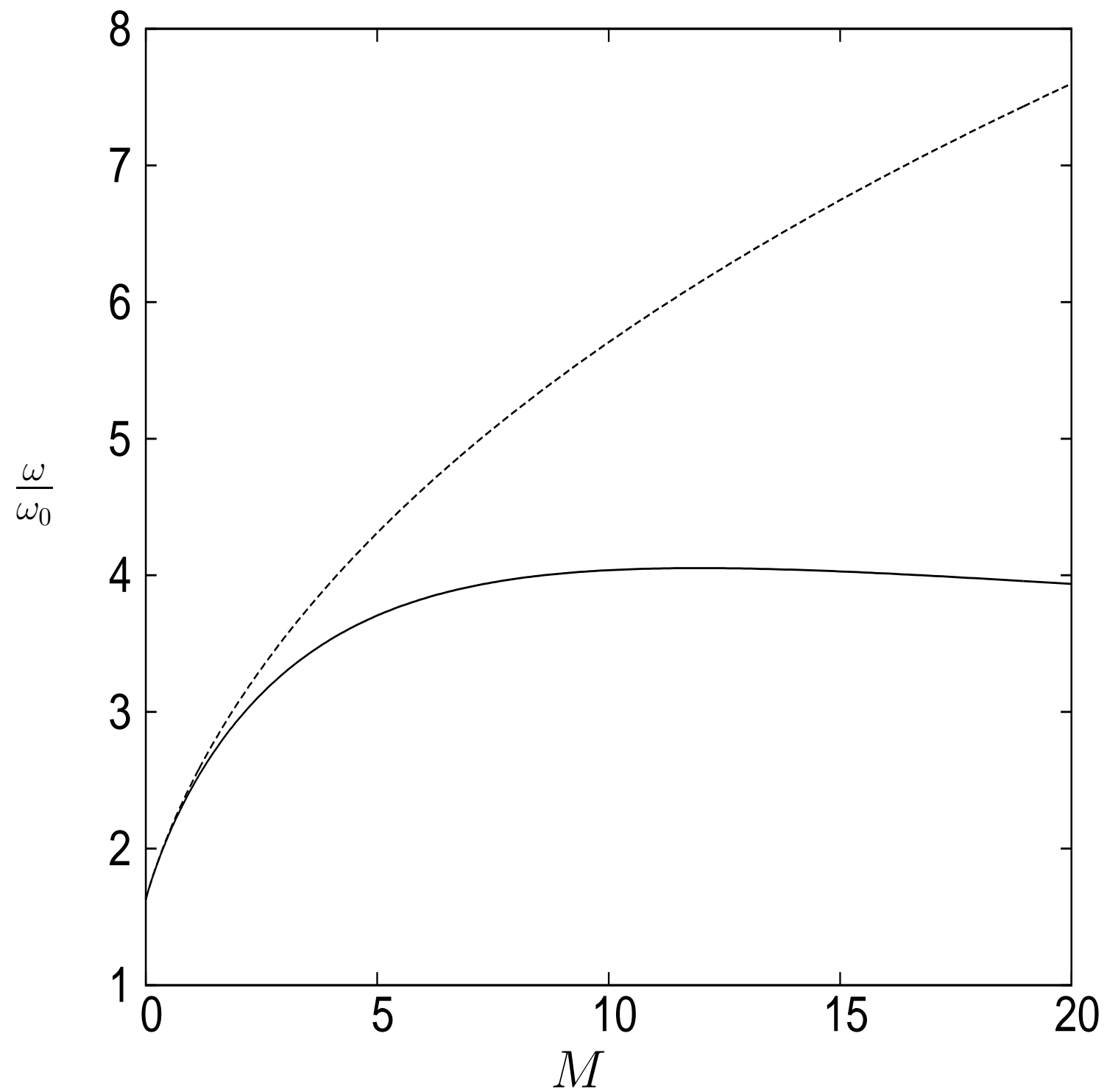

Figure $4 \mathrm{~b}$. Plot of $\omega_{A}(M)$ for the hyperbolic container with $c^{*}=1 \mathrm{~m}, H^{*}=0.5 \mathrm{~m}$ and $l=2.0 \mathrm{~m}$ for our non-hydrostatic pressure assumption (5.1) (solid line) and Cooker (1994)'s hydrostatic pressure assumption (5.6) (dashed line). 


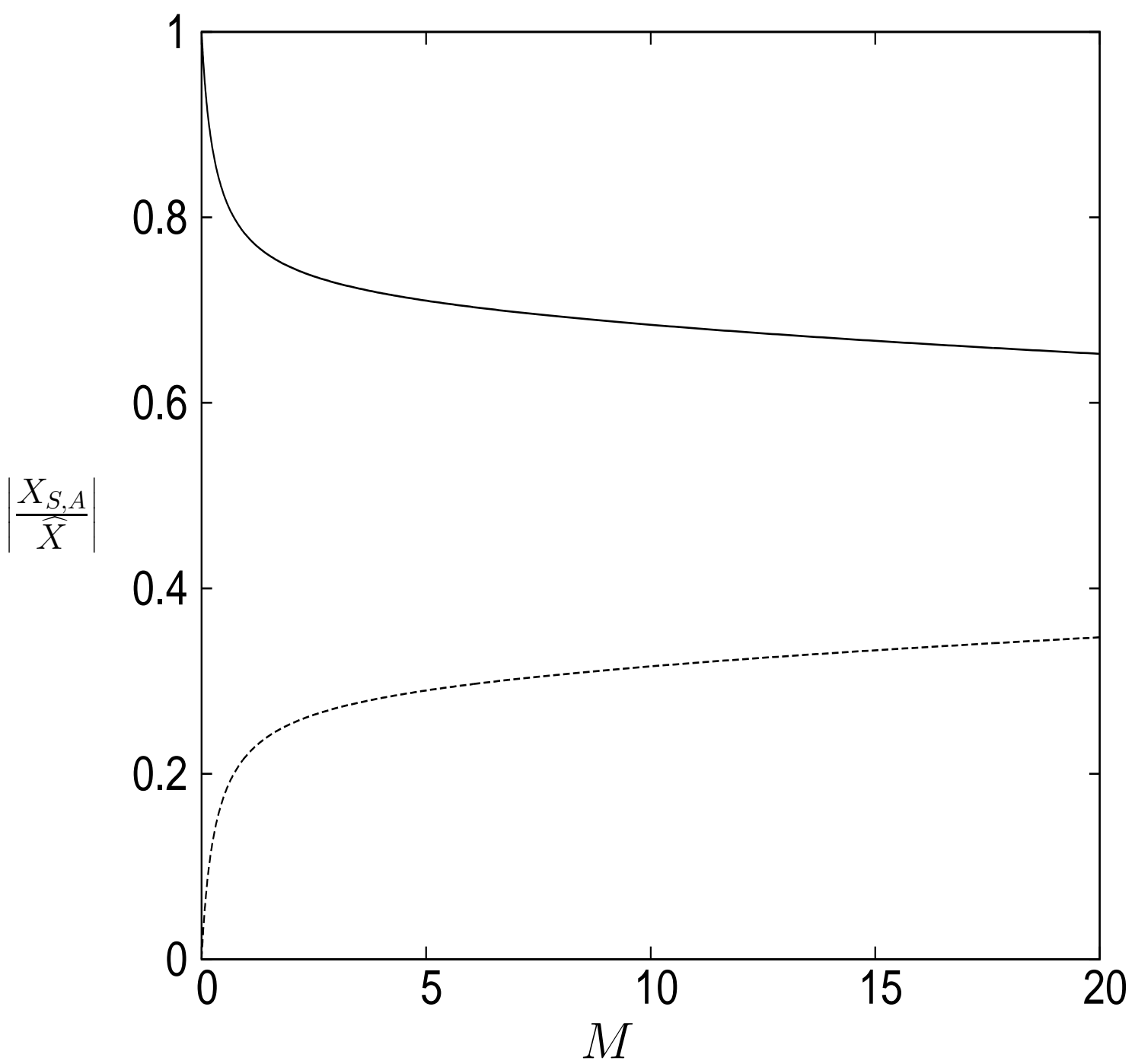

Figure 5a. Plot of $X_{S}(M)$ (solid line) and $X_{A}(M)$ (dashed line) for the hyperbolic container with $c^{*}=1.0 \mathrm{~m}, H^{*}=0.5 \mathrm{~m}$ and $l=2.0 \mathrm{~m}$ from figure $3 \mathrm{a}$. 


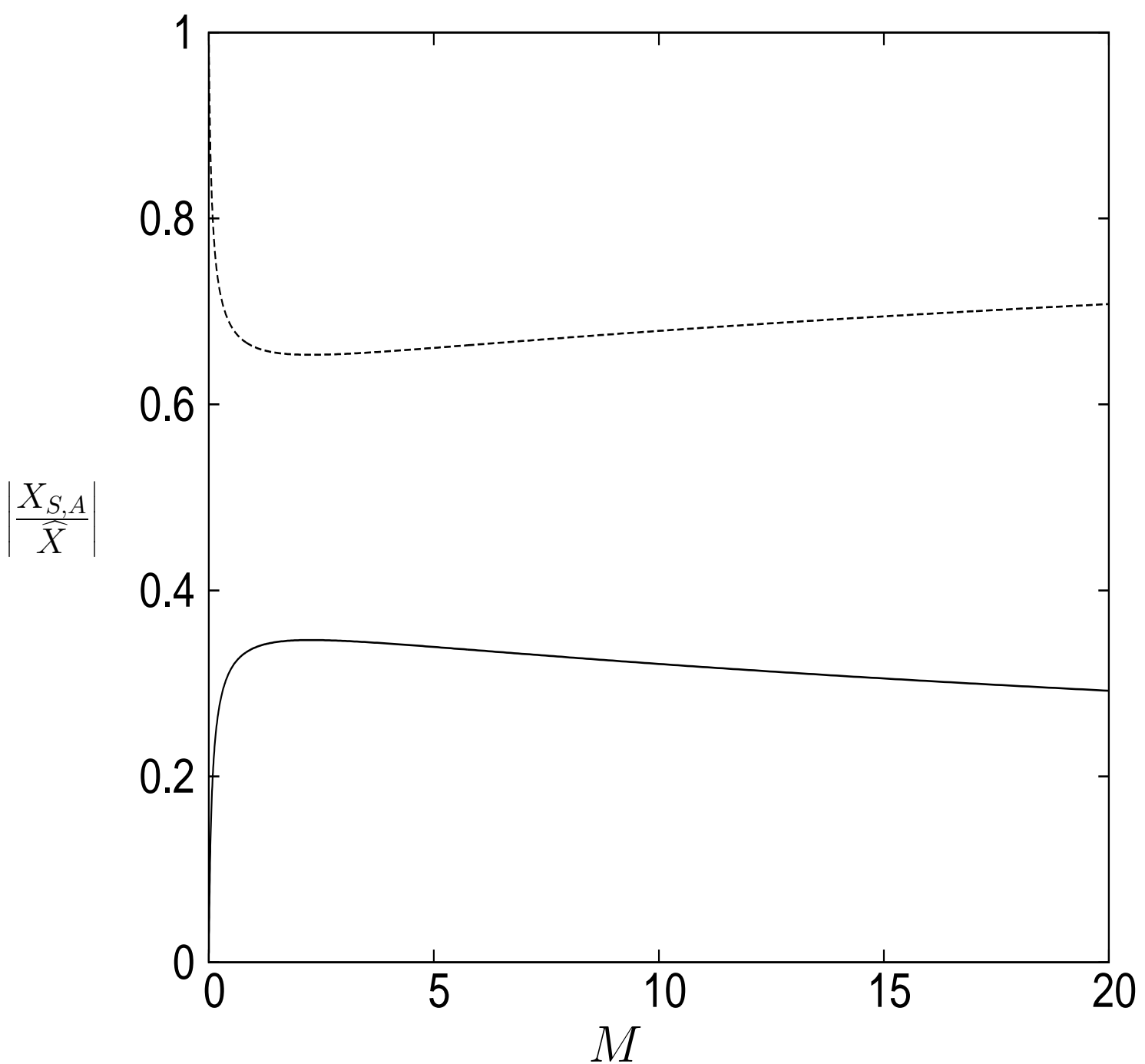

Figure 5b. Plot of $X_{S}(M)$ (solid line) and $X_{A}(M)$ (dashed line) for the hyperbolic container with $c^{*}=1.0 \mathrm{~m}, H^{*}=0.5 \mathrm{~m}$ and $l=0.5 \mathrm{~m}$ from figure $3 \mathrm{a}$. 


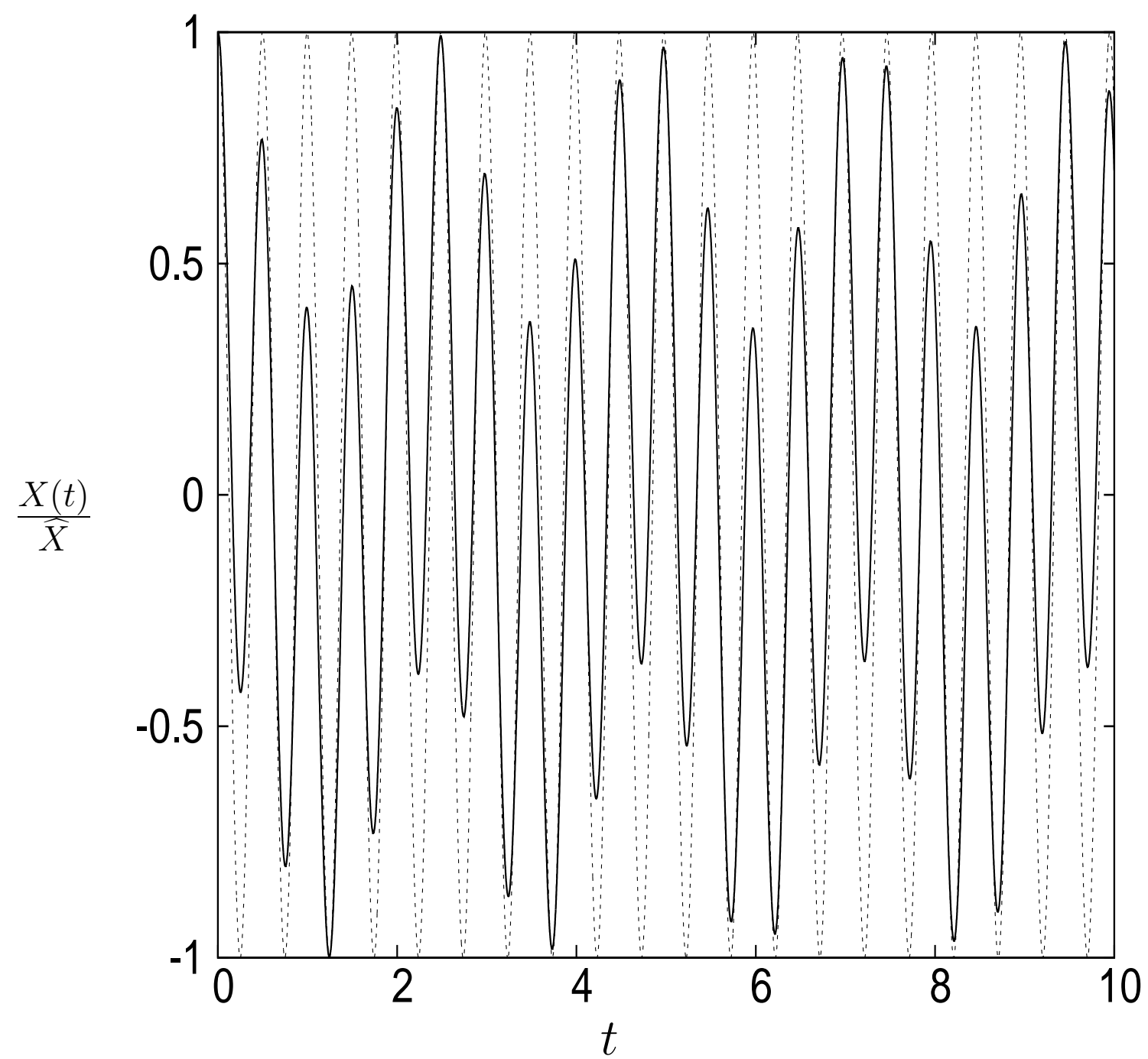

Figure 6. Plot of $X(t) / \widehat{X}$ (solid line) and $\cos \left(\omega_{A} t\right)$ (dashed line) for the hyperbolic container with $c^{*}=1.0 \mathrm{~m}, H^{*}=0.5 \mathrm{~m}, M=10.10$ and $l=0.5 \mathrm{~m}$. 


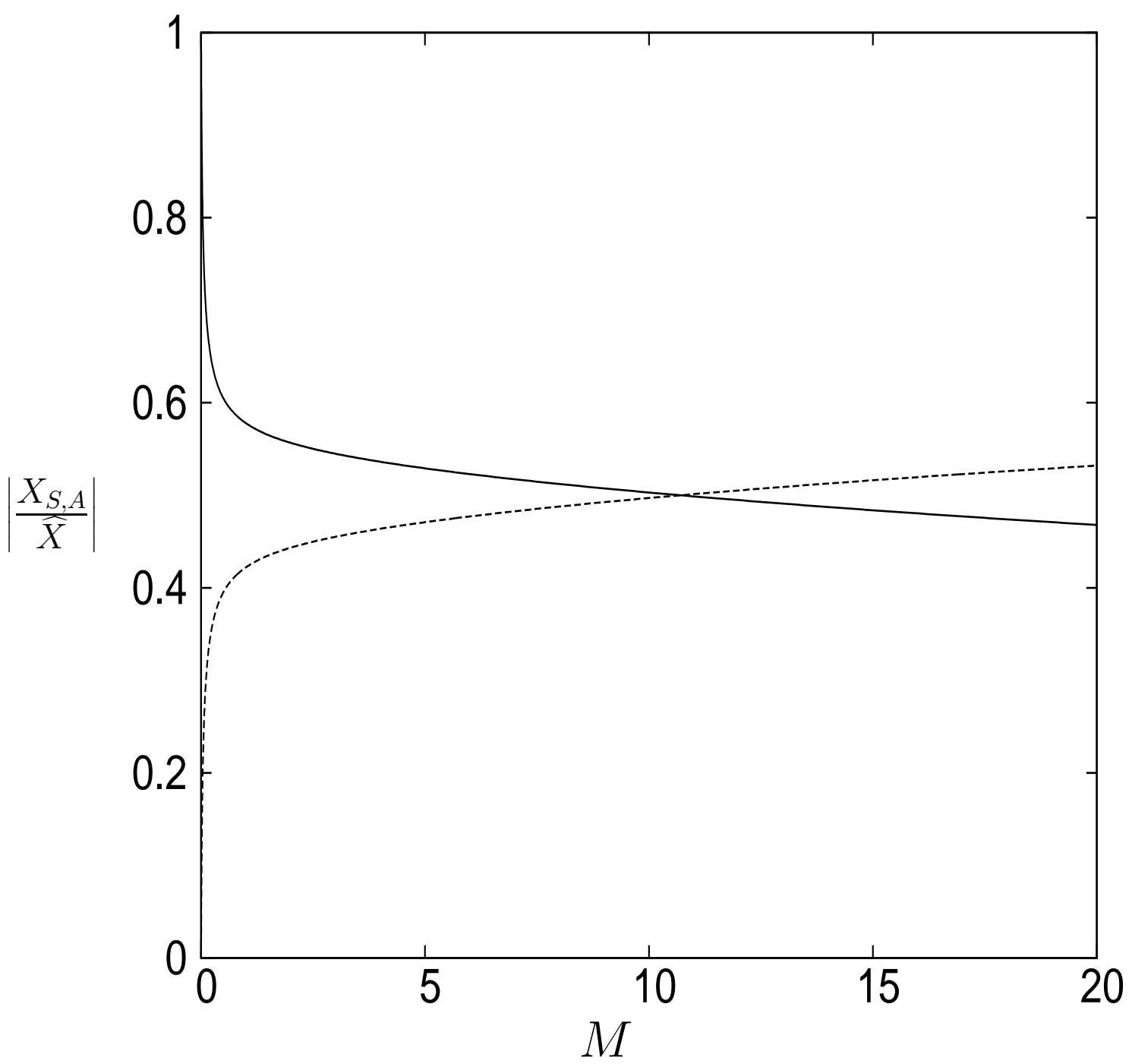

Figure 7. Plot of $X_{S}(M)$ (solid line) and $X_{A}(M)$ (dashed line) for the hyperbolic container with $c^{*}=1.0 \mathrm{~m}, H^{*}=0.5 \mathrm{~m}$ and $l=1.0 \mathrm{~m}$. 


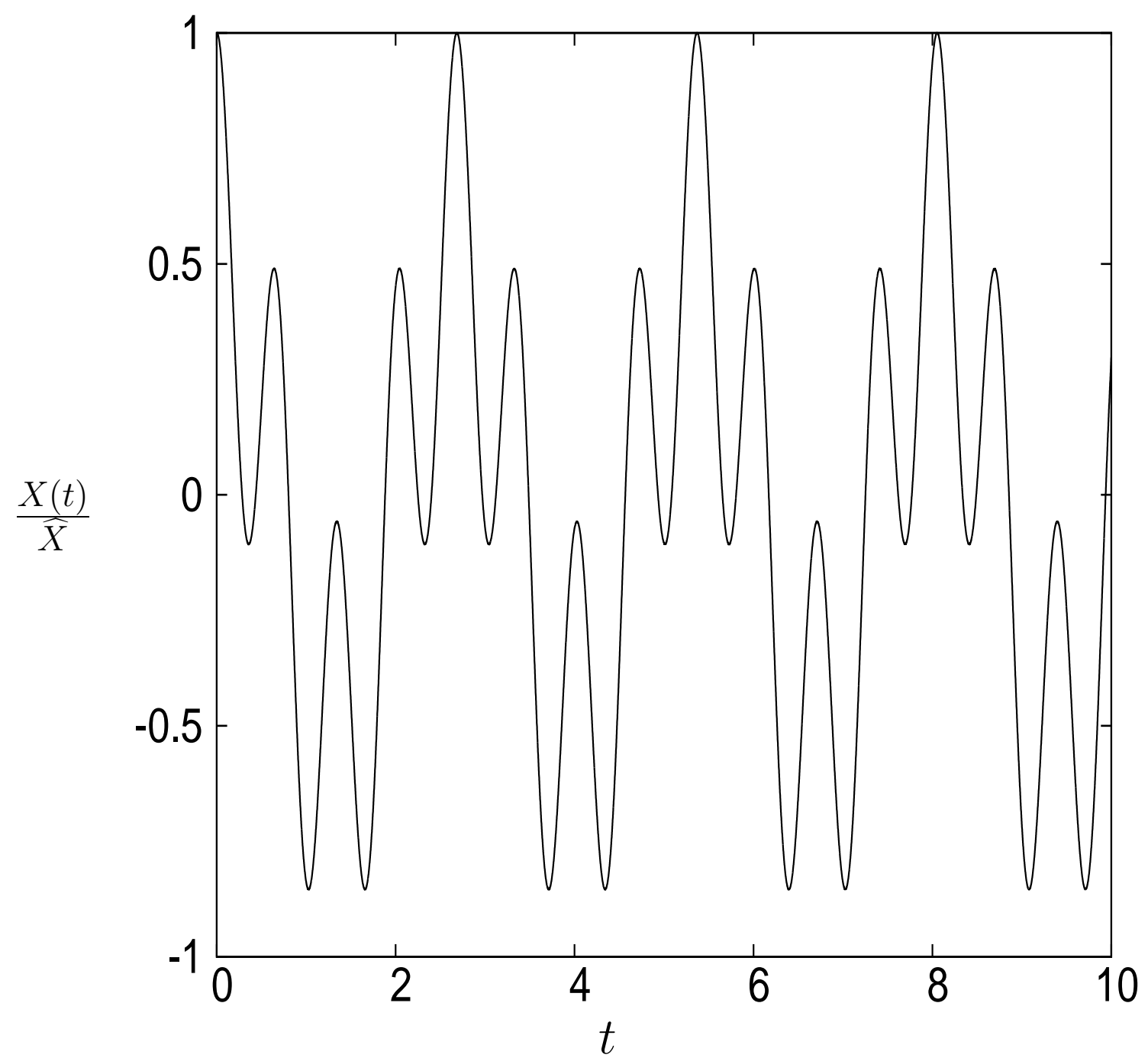

Figure 8. Plot of $X(t) / \widehat{X}$ for the hyperbolic container with $c^{*}=1.0 \mathrm{~m}, H^{*}=$ $0.5 \mathrm{~m}$ and $l=1.0 \mathrm{~m}$. 